\title{
Normal and abnormal gas patterns: which is which?
}

\author{
Deng-Wei Chou ${ }^{1,2} \cdot$ Yeun Tarl Fresner $\mathrm{Ng} \mathrm{Jao}^{1} \cdot$ Shu-Chen $\mathrm{Han}^{3}$
}

Received: 14 May 2015 / Accepted: 25 May 2015 / Published online: 9 June 2015

(C) SIMI 2015

\begin{abstract}
A 58-year-old woman without diabetes or other immunocompromising diseases presented to the emergency department with a fever and progressive deterioration of consciousness of 2 days duration. She had been bedridden for a month because of major depression. On examination, she was comatose, febrile, tachycardic, tachypneic, and hypotensive. Extensive bruising was present over the trunk, and she had an $8-\mathrm{cm}$ sacral pressure ulcer (Stage IV, The National Pressure Ulcer Advisory Panel). Neither cutaneous blisters nor crepitation was noted. The remainder of the physical examination was unremarkable. Laboratory examination showed a leukocytosis, thrombocytopenia, elevated C-reactive protein, and a prolonged coagulation time. A plain radiograph of the kidney ureter bladder (Fig. 1a) indicated gas formations over the soft tissues of the bilateral hips and the pelvic regions, which suggested
\end{abstract}

Shu-Chen Han

han520719@gmail.com

Deng-Wei Chou

choudw@gmail.com

Yeun Tarl Fresner Ng Jao

pogibomb@hotmail.com

1 Department of Critical Care Medicine, Tainan Municipal Hospital, Tainan, Taiwan, ROC

2 Department of Nursing, Chung Hwa University of Medical Technology, Tainan, Taiwan, ROC

3 Department of Radiology, Tainan Municipal Hospital, Tainan, Taiwan, ROC necrotizing soft tissue infections (NSTIs). These findings were confirmed through a computed tomography (CT) scan of the abdomen and pelvis (Fig. 1b-f). The diagnosis of NSTIs of the thighs, buttocks, and pelvis caused by a pressure ulcer was made. Broad-spectrum antibiotics and surgical debridement were performed immediately. NSTIs were confirmed through histopathologic examination.

A delay in diagnosis of NSTIs leads to delayed surgical debridement, and the patient's condition may deteriorate and result in death [1]. Clinical characteristics that facilitate the process of identifying NSTIs include: pain disproportionate to the appearance of the lesion, tense edema, skin discoloration, blisters, bullae, necrosis, and crepitus [2]. However, this patient was an exception to the rule because she was both comatose, and did not exhibit any cutaneous symptoms, rendering the diagnosis difficult. Diagnosing NSTIs in the gluteal area and pelvis is challenging when pathognomonic physical findings are not available, and a plain radiograph is sometimes valuable. This case highlights the importance of an early and accurate interpretation of a plain radiograph when the typical "normal" gas formations are not normally located or distributed. This specific finding may be missed even by experienced radiologists and emergency physicians, which decreases the patient's survival chances. These lesions might be incorrectly identified as bowel loops when cutaneous lesions are absent, missing the diagnosis. A CT scan should follow the plain radiograph, if one is able to think of the entity and institute a search for its presence, and to confirm the diagnosis and assess the extent of the NSTIs. A CT scan can show the spatial relationships between the NSTIs and the surrounding structures, and even reveal the routes of spread of this disease. This case is valuable because lesions in the thighs, buttocks, and pelvis were clearly identified, and the extension of the lesions into the 
Fig. 1 a Plain radiograph of the kidney ureter bladder, indicating gas collections over the soft tissue of the bilateral hips (arrows) and pelvis (arrowhead) regions. b CT scan of the abdomen and pelvis (coronal view), indicating abundant gas collection in the gluteus medius muscle (arrows), adductor muscles of the hip (curved arrow), and pelvis (arrowhead). Focal fluid collection can be observed within the iliacus muscle (asterisk). CT scan images (cross-sectional view) indicating $\mathbf{c}$ gas bubbles extending along the fascial plane from the sacral pressure ulcer (arrowhead) to the gluteus medius muscle (arrow), d gas bubbles extending into the pelvis from the gluteus medius muscle (arrow) through the piriformis muscle (arrowhead), and $\mathbf{e}$ gas bubbles extending into the iliopsoas muscle (arrowhead) from the gluteus medius muscle (arrow). Osteomyelitis in the left ilium (curved arrow) is shown, $\mathbf{f}$ gasforming abscesses are observed within the iliopsoas muscle (arrows)
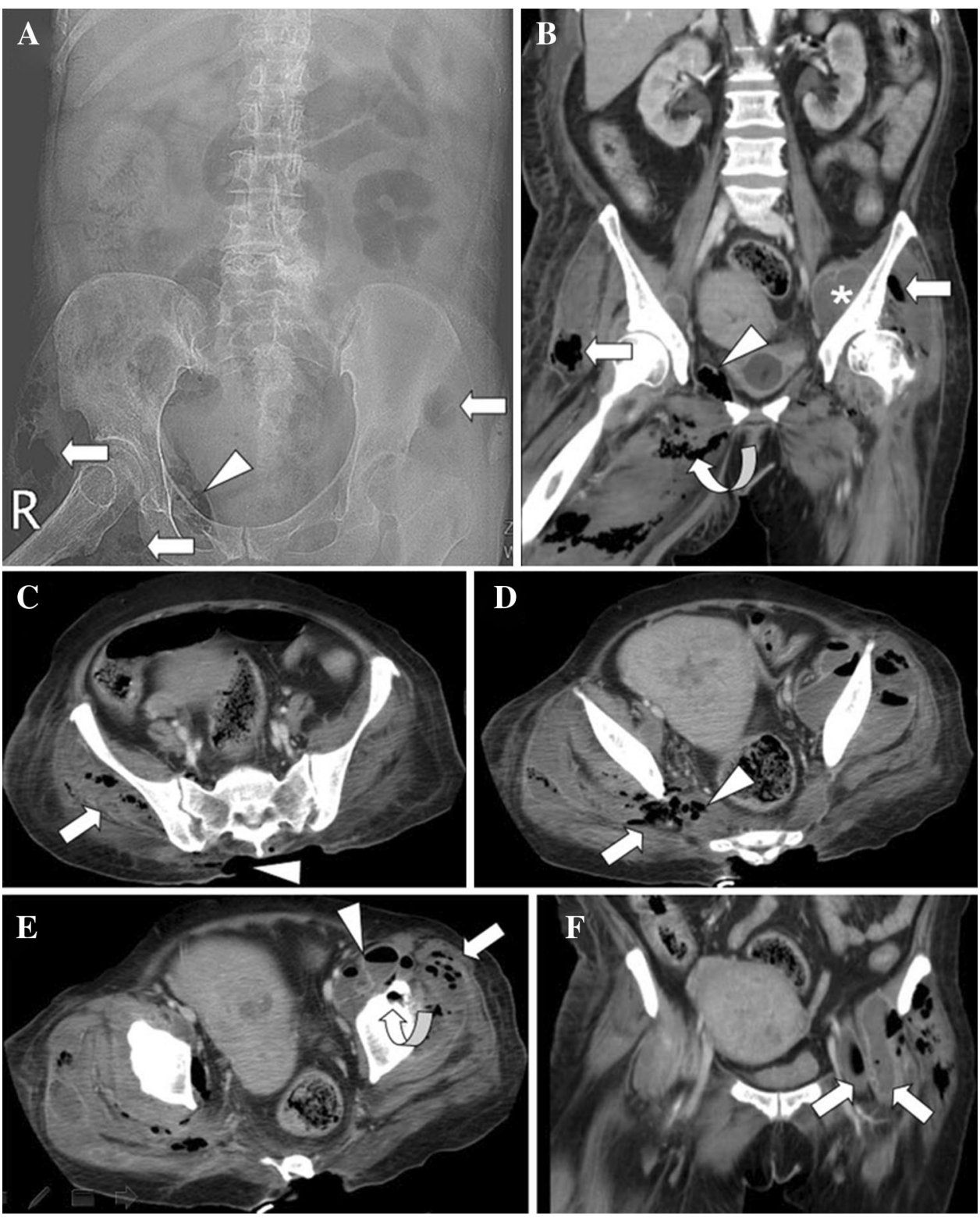

pelvis through the piriformis, gluteus medius, and iliopsoas muscles was shown.

Conflict of interest The Authors declare that they have no conflict of interest.

Statement of human and animal rights All procedures performed in studies involving human participants were in accordance with the ethical standards of the institutional and/or national research committee and with the 1964 Helsinki declaration and its later amendments or comparable ethical standards. This article does not contain any studies with human and animals performed by any of the authors.
Informed consent None.

\section{References}

1. Anaya DA, Dellinger EP (2007) Necrotizing soft-tissue infection: diagnosis and management. Clin Infect Dis 44(5):705-710

2. Mullangi PK, Khardori NM (2012) Necrotizing soft-tissue infections. Med Clin North Am 96(6):1193-1202 